\title{
ESTUDIO DE LOS FORAMINÍFEROS DE LA FORMACIÓN PIRABAS (MIOCENO INFERIOR), ESTADO DE PARÁ, BRASIL Y SU CORRELACIÓN CON FAUNAS DEL CARIBE
}

\author{
Vladimir de Araújo Távora \& Jane Maria Garrafielo Fernandes \\ Departamento de Geologia, Centro de Geociências, Universidade Federal do Pará, \\ Caixa Postal 1611, Belém-Pa, Brasil, CEP 66075-900.
}

(Recibido 23/2/1999; Aceptado 27/4/1999)

\begin{abstract}
Vertical variation in the foraminifera content detected by quantitative and qualitative studies permitted recognition of eight biofacies along the Pirabas Formation at the investigated area. The assemblages are related to shallow shelf and marginal marine paleoenvironments. Cyclical fluctuations in the marginal marine environments demonstrated changes in the water's depth and salinity.

The presence of planktonic foraminifera confirms the Early Miocene age for the Pirabas Formation. The presence of Globorotalia kugleri Bolli and Globigerinoides primordius Blow \& Banner at the base, and Globigerinoides altiaperturus Bolli and Globigerinoides trilobus trilobus (Reuss) at the top of outcrop permit correlation to N4 and N5 International Biostratigraphic Zones of Blow (1969).
\end{abstract}

RESUMEN: Las variaciones verticales en el contenido de las asociaciones de foraminíferos, detectadas por medio de estudios cuantitativos y cualitativos, permitieron el reconocimiento de ocho biofacies en la Formación Pirabas, correspondientes a los subambientes de plataforma somera y lagunar. Los sedimentos fueron depositados en las áreas lagunares en función de las variaciones en la profundidad y la salinidad.

La presencia de los foraminíferos planctónicos confirma la edad Mioceno Inferior para la Formación Pirabas. El registro de Globorotalia kugleri Bolli y Globigerinoides primordius Blow \& Banner en la base y Globigerinoides altiaperturus Bolli y Globigerinoides trilobus trilobus (Reuss) en el tope del afloramiento puede ser correlacionado con las Zonas Bioestratigráficas Internacionales N4 y N5 de Blow (1969)

\section{INTRODUCCIÓN}

La Formación Pirabas (Maury, 1925) es una importante unidad litoestratigráfica del norte de Brasil y revela un rico y variado contenido fosilífero marino del Neógeno. Esta Formación, con presencia discontinua en los estados de $\mathrm{Pa}-$ rá, Maranhão y Piauí, litológicamente está constituida por rocas calcáreas de edad Mioceno
Inferior y caracteriza un ambiente marino de aguas someras y cálidas.

La estrecha relación entre la Formación Pirabas y las diferentes formaciones del hemisferio norte, demostrada por el gran número de especies en común, llevó a Maury (op.cit.) a admitir una importante correspondencia con las formaciones Chipola y Baitoa en Santo Domingo, ambas del Mioceno Inferior (Ferreira, 1980). El género Orthaulax, con pocas especies distribuidas 
en las formaciones pertenecientes al intervalo Oligoceno Superior-Mioceno Inferior, en el lado atlántico posee una distribución geográfica limitada entre el Estado de Georgia (Estados Unidos) y los estratos de la Formación Pirabas en la cuenca de Barreirinhas, estado de Maranhão. Basado en la presencia del referido género de gastrópodo, que constituye el fósil guía mas importante encontrado en estos estratos, la Formación Pirabas es correlacionada con las formaciones Tampa y Chipola, ambas de la península de La Florida en los Estados Unidos (Ferreira, op.cit.).

Con el objetivo de contribuir al conocimiento de la Formación Pirabas se realizó en este trabajo un estudio sobre los foraminíferos de un afloramiento situado en la localidad de Aricuru. Este afloramiento se presenta como un acantilado de 3 metros de altura y 50 metros de extensión aproximadamente y está localizado en el litoral del estado de Pará, a $2 \mathrm{~km}$ al NNO de la ciudad de Maracanã (Fig. 1).

Esta localidad geológica se caracteriza por dos litotipos principales: arcillas, que constituyen el tercio inferior del perfil (capas 1 y 2) y rocas carbonáticas arenosas en los tercios medio y superior (a partir de la capa 3). Fueron individualizadas 10 horizontes, con espesores que varían entre 5 y $80 \mathrm{~cm}$.

En términos de macrofósiles fue registrada la presencia de corales, briozoos (dominantemente Steginoporella pirabensis Barbosa), espinas de equinodermos, bivalvos (familias Pectinidae, Plicatulidae y Ostreidae), icnofósiles (principalmente Thalassinoides icnospp.), macroforaminíferos (familia Soritidae), un fragmento de una costilla de sirenideo y fragmentos vegetales.

El total de material de las muestras de cada nivel (20 g de sedimento) fue procesado para la investigación de foraminíferos, ostrácodos y briozoos, por medio de métodos usuales de pesaje, desintegración mecánica, lavado, tamizado en 5 fracciones granulométricas $(0,500 \mathrm{~mm}, 0,250 \mathrm{~mm}$, $0,125 \mathrm{~mm}, 0,074 \mathrm{~mm}$ y $0,062 \mathrm{~mm})$, secado y recolección de los foraminíferos. También fueron colectados $5 \mathrm{~g}$ de sedimento para estudios de nannoplancton calcáreo, cuyo trabajo aún se encuentra en la fase de preparación de las láminas para una futura publicación de los resultados obtenidos.
Junto con los foraminíferos, concentrados en una gran mayoría en las fracciones $0,250 \mathrm{~mm}$ y $0,125 \mathrm{~mm}$, se encontró una abundante fauna de ostrácodos y briozoos. En la fracción mayor aún se registraron bivalvos, espinas de equinodermos, fragmentos de vegetales y macroforaminíferos, repitiéndose prácticamente la misma fauna encontrada macroscópicamente.

La clasificación sistemática adoptada en este trabajo para los foraminíferos fue basada en los criterios empleados por Loeblich \& Tappan (1964), Blow (1969), Stainforth et al. (1975), así como en la vasta bibliografía complementaria específica.

El estudio cuantitativo está regido por los parámetros ecológicos índice de diversidad Alfa de Fisher y predominacia genérica y específica. Para este fin todas las muestras fueron consideradas, ya que todas ellas poseen representatividad numérica superior a 100 individuos.

\section{ANÁLISIS MICROFAUNÍSTICO}

El número de foraminíferos se muestra bastante homogéneo en las muestras estudiadas, presentando en su mayoria caparazones completos, en excelente estado de preservación. El promedio de los ejemplares por capa es de aproximadamente en 200 individuos. Los niveles que mostraron valores diferentes fueran las capas 4,6 y 8 que contenían un poco mas de 100 individuos.

Considerando los foraminíferos, generalmente pequeños, predominan los rotalídeos bentónicos, seguidos por los miliolídeos. Los rotalídeos plantónicos y los textularídeos están poco representados, presentándose como un grupo insignificante en relación a los demás.

Analizando las formas bentónicas (Fig. 2) fueron reconocidas:

\section{Ammonia beccarii var.parkinsoniana} (d'Orbigny)

A. beccarii var.sobrina (Shupack)

A. beccarii var. tepida (Cushman)

Amphistegina lessonii (d'Orbigny)

Archaias angulata (Fichtel \& Moll) 


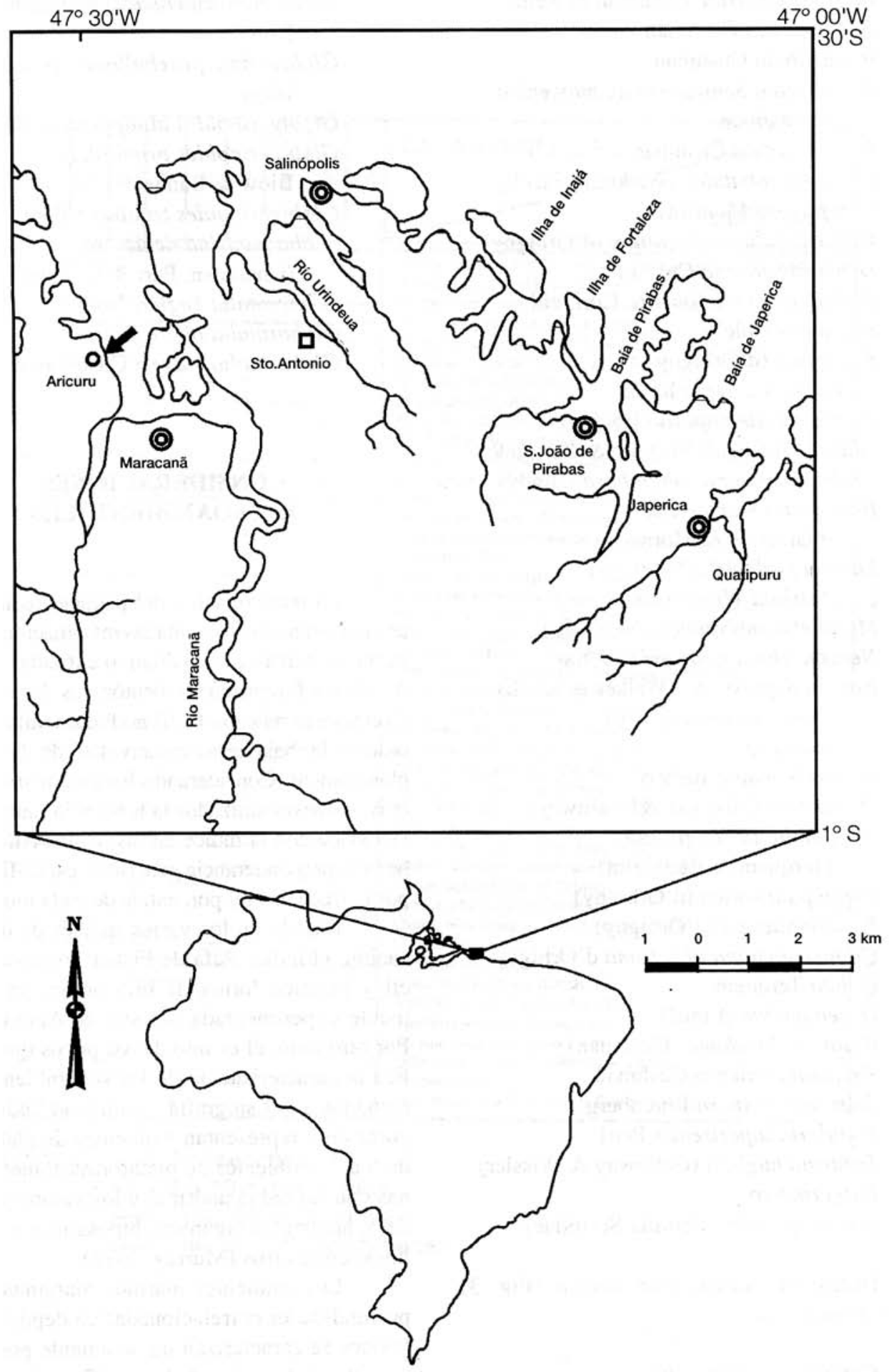

Fig. 1: Mapa de ubicación del área de estudio. 
Bolivina caudriae Cushman \& Renz

B. marginata Cushman

B. plicatella Cushman

B. scalprata Schwager var. miocenica Macfadyen

B. cf. striatula Cushman

Cibicides lobatulus (Walker \& Jacob)

C. refulgens Montfort

Criboelphidium poeyanum (d'Orbigny)

Discorbis advena Cushman

Elphidium galvestonense Kornfeld

E. gunteri Cole

E. sagrum (d'Orbigny)

Fissurina laevigata Reuss

Florilus grateloupi (d’Orbigny)

Glabratella sigali Sienglie \& Bermudez

Globocassidulina subglobosa (Brady)

Hanzawaia mantaensis

(Galloway \& Morrey)

Lagena perlucida (Montagu)

L. substriata Williamson

Miolinella subrotunda (Montagu)

Neoconorbina terquemi (Rzehak)

Nonion depressulus (Walker \& Jacob)

Nonionella pirabensis Petri

Nodosaria sp.

Oolina leoniana Bertels

O. nuttalli (Galloway \&Heninway)

Ophtalmidium longiscata

(Terquem \& Berthleim)

Pyrgo patagonica (d'Orbigny)

$P$. subsphaerica (d'Orbigny)

Quinqueloculina akneriana d'Orbigny

Q. lata Terquem

Q. seminulum (Linné)

Rosalina floridana (Cushman)

Siphonina pulchra Cushman

Spirillina vivipara Ehrenberg

Textularia japerisensis Petri

Trifarina hughesi (Galloway \& Wissler)

Uvigerina sp.

gen. et sp. indet. (familia Soritidae)

Dentro las formas planctónicas (Fig. 3) fueron reconocidas:

Catapsydrax dissimilis

(Cushman \& Bermudez)
Globigerina ciperoensis angustiumbilicata Bolli

Globigerina. praebulloides praebulloides Blow

Globigerinoides altiaperturus Bolli

Globigerinoides primordius

Blow \& Banner

Globigerinoides trilobus trilobus (Reuss)

Globoquadrina dehiscens

(Chapman, Parr \& Collins)

Globorotalia kugleri Bolli

Globorotalia obesa Bolli

Globorotalia mayeri Cushman \& Ellisor

\section{CONSIDERACIONES PALEOAMBIENTALES}

La interpretación del paquete sedimentario se hizo tomando en cuenta las informaciones de carácter cuantitativo y cualitativo extraídas del estudio de los foraminíferos bentónicos. Los datos referentes a la relación PL/B no fueron utilizados debido a la baja representatividad de las formas planctónicas. Considerando los parámetros cuantitativos fueron utilizados la tendencia faunística relacionada con el índice de diversidad Alfa de Fisher y la preponderancia genérica y específica dadas por la frecuencia y porcentaje de cada muestra.

Basado en los varios índices de diversificación, el índice Alfa de Fisher presenta una fácil y práctica forma de utilización, ya ampliamente experimentada (Moura \& Abreu, 1980). Por otro lado, él es uno de los pocos que cuantifica la caracterización de los subambientes relacionados a la fisiografía y salinidad. índices mayores de 7 representan ambientes de plataforma; de 5 a 7, ambientes de plataforma someros/lagunas con salinidad anormal y los valores menores de 5, ambientes lagunares hiposalinos e hipersalinos, entre otros (Murray, 1973).

Los ambientes marinos marginales y sus profundidades correlacionadas en depósitos geológicos se caracterizan no solamente por los bajos valores de diversidad específica, sino también por los altos valores de dominancia de las 

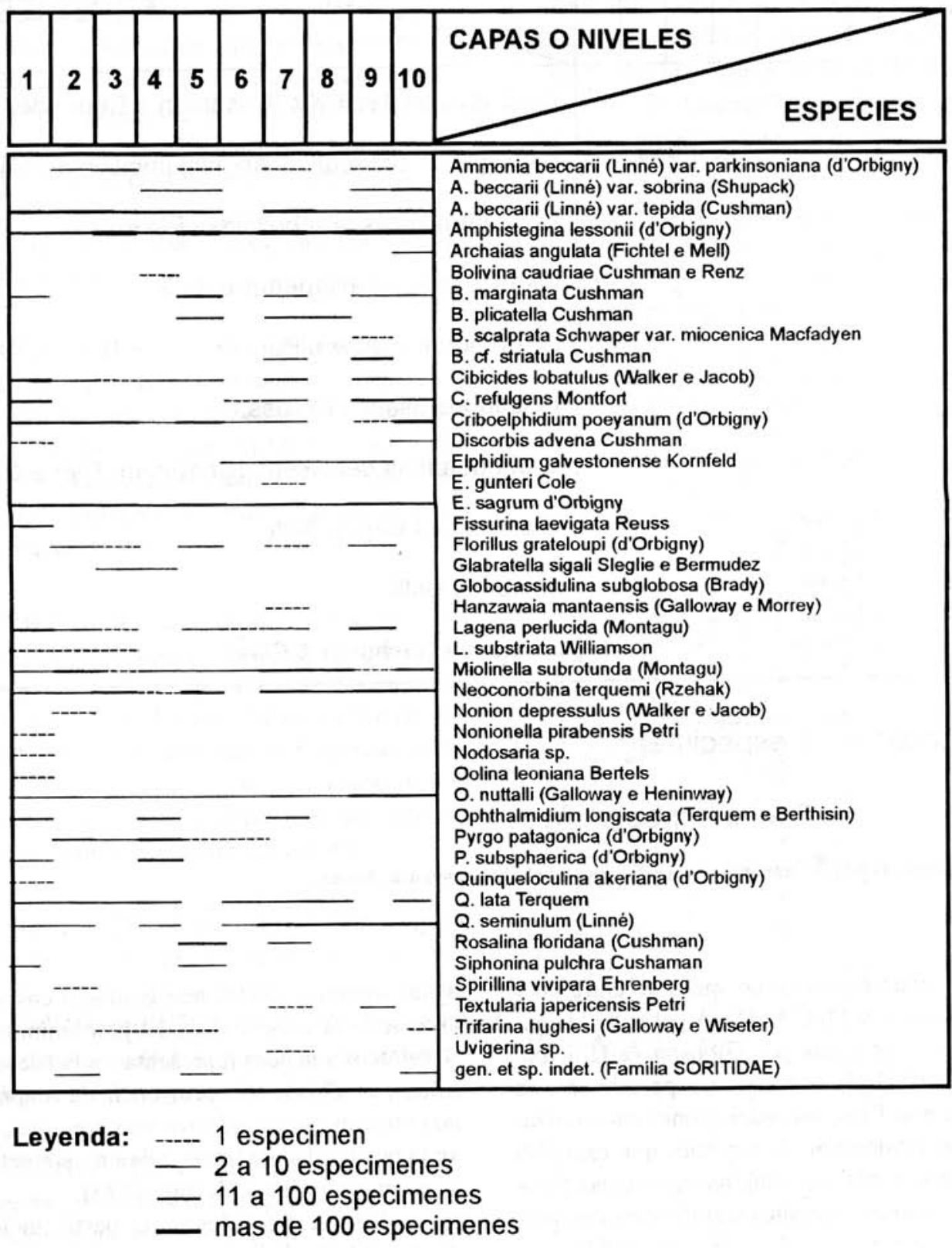

Fig. 2: Distribución de los foraminíferos bentónicos en el afloramiento de Aricuru. 


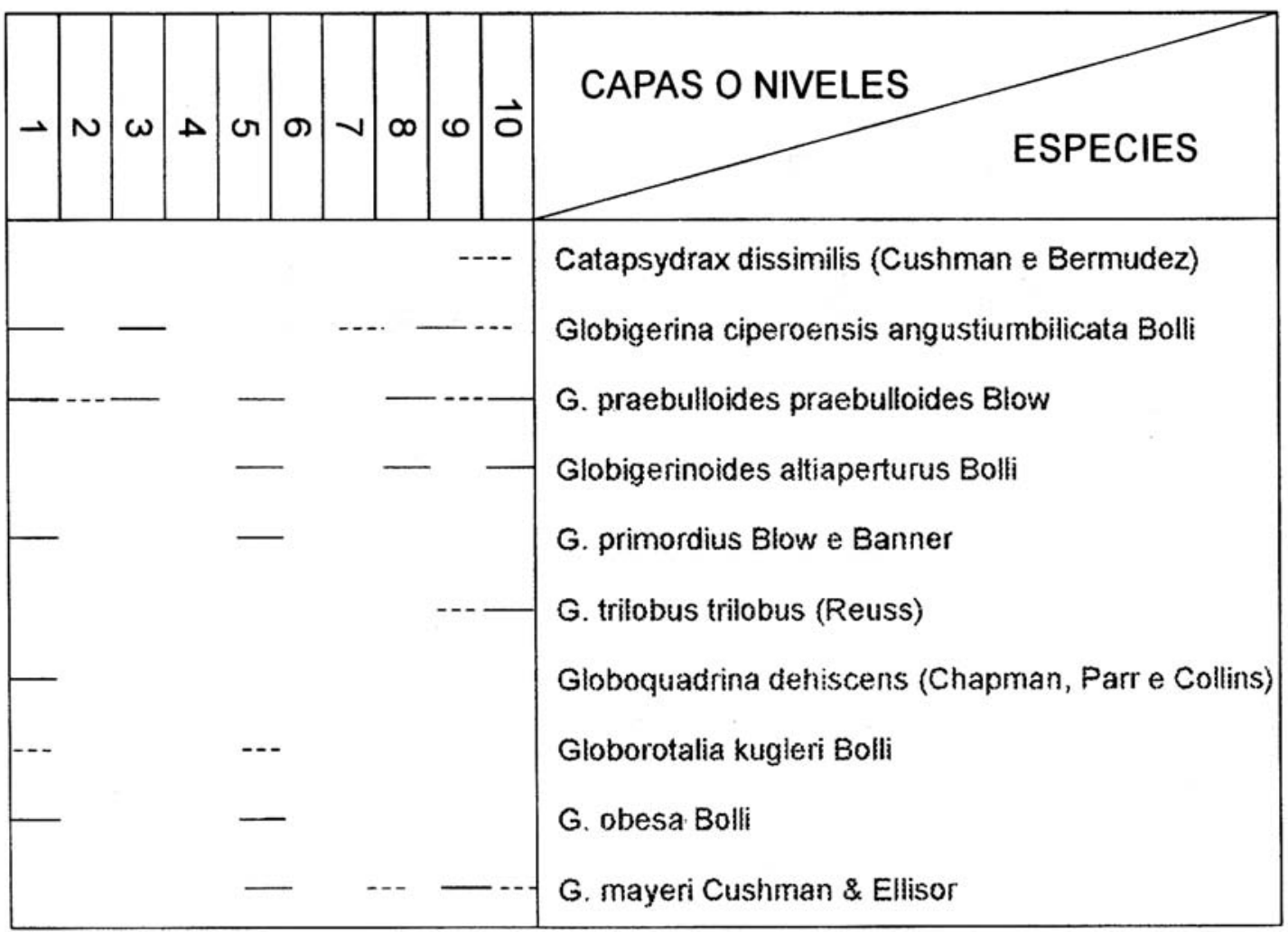

Leyenda: $\cdots 1$ especimen

\section{- 2 a 10 especimenes}

Fig. 3: Distribución de los foraminíferos planctónicos en el afloramiento de Aricuru.

especies, cuantificada como variando entre $30 \mathrm{y}$ 95\% (Gibbson \& Hill, 1992). A pesar de las excepciones constatadas por Gibbson \& Hill (op. cit.) fuera del Golfo de México, especialmente en las aguas mas frías, las asociaciones caracterizadas por el predominio de especies que excedeN al 35\% de la población total, han sido generalizadas como correspondientes a ambientes con profundidades inferiores a $3 \mathrm{~m}$ (Walton, 1964).

El análisis cualitativo del conjunto faunístico muestra una monotona microfauna de foraminíferos. Los generos Ammonia, Amphistegina y Elphidium/Criboelphidium, todos típicos de aguas someras, son los más comunes en todas las asociaciones encontradas. El predominio de los Rotalídeos y la poca representatividad de textularideos, asociados a la ocurrencia de Amphistegina como uno de los géneros mas comunes, excluye la posibilidad de tratarse de un ambiente transicional hiposalino (Murray, 1973).

Fueron obtenidos en el perfil, índices de diversidad alfa de Fisher que variaron entre 3 y 7 , permitiendo caracterizar en toda la sección estudiada, la mayor incidencia de los ambientes marinos marginales, entre los que se destacan los lagunares con salinidad anormal (Fig. 4). 
La predominancia expresiva de Amphistegina lessonii (d'Orbigny), con valores superiores a $35 \%$ (porcentajes variando entre $43 \%$ y $57 \%$ en los niveles $5,6,7,8,9$ ), permite sugerir que los sedimentos se depositaron en ambientes de poca profundidad, posiblemente inferior a los 3 metros.

Basado en los datos referentes a la tendencia faunística, principalmente en relación a los indices de diversidad Alfa de Fisher, y en menor importancia por la Dominancia Genérica y Específica, fue posible observar las alteraciones en las asociaciones registradas, correspondientes a las diferentes biofacies.

BIOFACIES 1 (correspondiente al nivel 1): géneros mas comunes: Ammonia, Amphistegina y Elphidium/Criboelphidium; dominancia de Ammonia beccarii (Linné) (16\%); índice de diversidad Alfa correspondiente a 7, presencia de icnofósiles; ambiente inferido: marino somero con salinidad normal.

BIOFACIES 2 (correspondiente al nivel 2) géneros mas comunes: Ammonia, Amphistegina y Elphidium/Criboelphidium; dominancia de Ammonia beccarii (Linné) (24\%); índice de diversidad Alfa correpondiente a 5; presencia de bivalvos, espinas de equinodermos, icnofósiles y macroforaminíferos; ambiente inferido: marino somero o lagunar con salinidad normal.

BIOFACIES 3 (correspondiente al nivel 3) géneros mas comunes: Amphistegina, Ammonia y Elphidium; dominancia de Amphistegina lessonii (d'Orbigny) (20\%); índice de diversidad Alfa correpondiente a 4; presencia de bivalvos; ambiente inferido: lagunar hipersalino.

BIOFACIES 4 (correspondiente al nivel 4) géneros mas comunes: Amphistegina, Ammonia y Criboelphidium; dominancia de Amphistegina lessonii (d'Orbigny) (27\%); índice de diversidad Alfa correpondiente a 6; presencia de bivalvos y gastrópodos; ambiente inferido: plataforma somera/lagunar con salinidad normal.
BIOFACIES 5 (correspondiente al nivel 5-6) géneros mas comunes: Amphistegina, Ammonia y Criboelphidium; dominancia de Amphistegina lessonii (d'Orbigny) (27\%); índice de diversidad Alfa correpondiente al intervalo 3-4; presencia de icnofósiles, macroforaminíferos, bivalvos, briozoos y espinas de equinodermos; ambiente inferido: lagunar hipersalino con profundidad probablemente inferior a $3 \mathrm{~m}$.

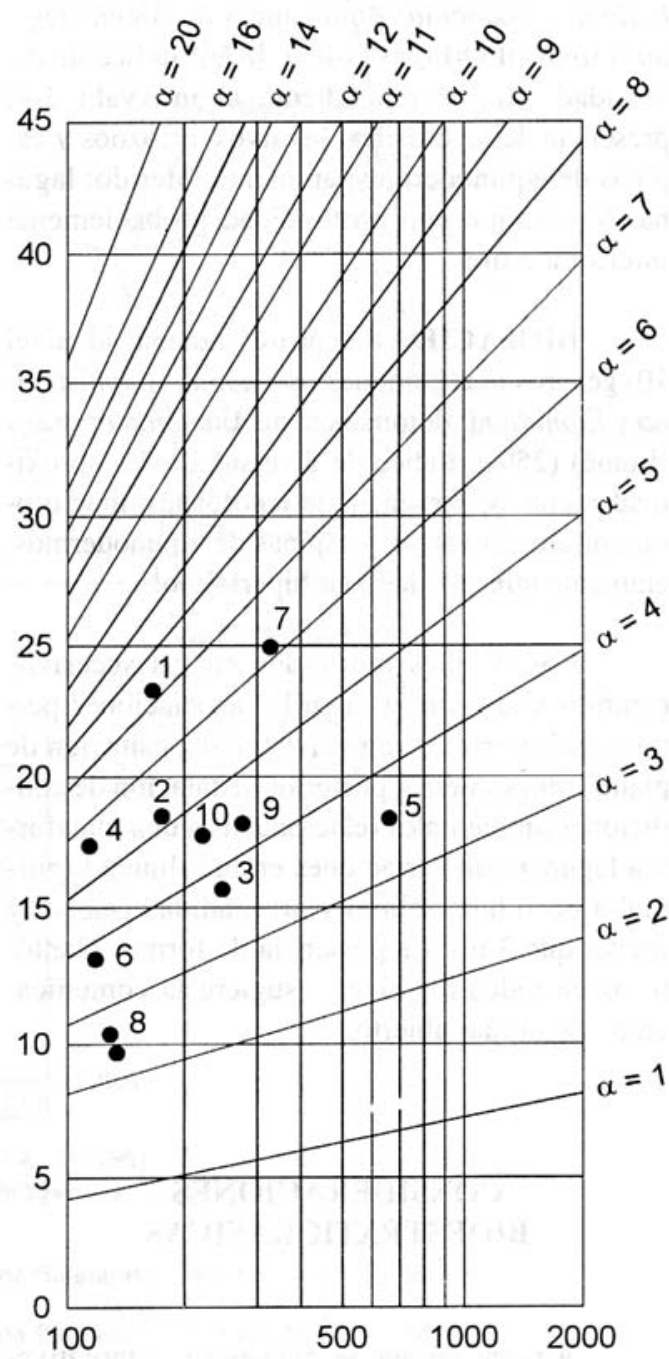

Fig. 4: Índices de diversidad Alfa de Fisher obtenidos en las capas del afloramineto de Aricuru. 
BIOFACIES 6 (correspondiente al nivel 7) géneros mas comunes: Amphistegina, Elphidium y Ammonia; dominancia de Amphistegina lessonii (d'Orbigny) (47\%);índice de diversidad Alfa correpondiente a 6; presencia de briozoos y espinas de equinodermos; ambiente inferido: plataforma somera/lagunar con salinidad normal y profundidad supuestamente menor a $3 \mathrm{~m}$.

BIOFACIES 7 (correspondiente al nivel 8 y 9) géneros mas comunes: Amphistegina, Elphidium y Ammonia; dominancia de Amphistegina lesonii (d'Orbigny) (44 a 48\%); índice de diversidad Alfa correpondiente al intervalo 3-4; presencia de icnofósiles, bivalvos, briozoos y espinas de equinodermos; ambiente inferido: lagunar hipersalino con profundidad probablemente inferior a $3 \mathrm{~m}$.

BIOFACIES 8 (correspondiente al nivel 10) géneros mas comunes: Ammonia, Amphistegina y Elphidium; dominancia de Ammonia beccarii (Linné) (25\%); índice de diversidad Alfa aproximadamente 4; presencia de icnofósiles, macroforaminíferos, bivalvos y espinas de equinodermos; ambiente inferido: lagunar hipersalino.

Los detalles mostrados en los sucesivos cambios que ocurrieron en las asociaciones permitieron inferir inicialmente una depositación de plataforma somera y posterior instalación de condiciones ambientales relacionadas a una plataforma lagunar con variaciones en la salinidad (normal a poco hipersalina) y profundidad (menor y mayor que $3 \mathrm{~m}$ ). La presencia de formas plantónicas en todos los niveles sugiere la comunicación con el mar abierto.

\section{CONSIDERACIONES BIOESTRATIGRÁFICAS}

A pesar de que se encuentra cuantitativamente poco representada, la microfauna de los foraminíferos plantónicos está constituida por especies de gran importancia bioestratigráfica. Los datos de aparecimiento y/o extinción de estas especies posibilitaron realizar algunas consideraciones sobre la edad de la depositación de los sedimentos estudiados (Fernandes, 1988; Fernandes \& Távora, 1990).

Basado en los datos obtenidos en este trabajo se correlaciona la porción basal del afloramiento (capa 1) con la zona N4 de Blow (1969) por el registro de Globorotalia kugleri Bolli y Globigerinoides primordius Blow \& Banner; la capa 5 con el límite entre las zonas N4 y N5 de Blow (op. cit.) debido a la coexistencia de Globorotalia kugleri Bolli, Globigerinoides primordius Blow \& Banner y Globigerinoides altiaperturus Bolli. También basado en la ocurrencia de Globigerinoides altiaperturus Bolli y Globigerinoides trilobus trilobus (Reuss) se correlacionan los niveles superiores del afloramiento (a partir de la capa 8) con la zona N5 de Blow (op. cit.).

Estudios anteriores (Fernandes, 1988) correlacionaron los sedimentos de subsuperficie de la Formación Pirabas de la región Bragantina con la zona N4 y los sedimentos de subsuperficie de la región urbana de Belém con la zona N5 de Blow (op.cit.). Posteriormente Fernandes \& Távora (1990) reconocieron la zona N5 también en la región Bragantina.

Estos datos nuevos caracterizan la presencia de las zonas N4 y N5 en el litoral paraense, ampliando asi la ocurrencia espacial de estas zonas en la área de la Formación Pirabas en el estado de Pará.

Reuniendo todos los datos bioestratigráficos recogidos para la Formación Pirabas hasta el presente y los datos obtenidos en este trabajo es posible realizar una correlación bioestratigráfica entre los puntos ya estudiados, procurando trazar las lineas temporales de la depositación de sedimentos en la cuenca de Pirabas, entre Belém, la zona Bragantina y la región del litoral del Estado de Pará (Fig. 5).

De esta manera, se define la zona N4 de Blow (1969) en la base del afloramiento de Aricuru (capas 1 a 5) en la zona del litoral Paraense y en el nivel 33.30m del pozo CB-UFPa-P1 (85), localizado a $5 \mathrm{~km}$ al NNO de la ciudad de Capanema, zona Bragantina. Por otro lado, la zona N5 está caracterizada en el tope del afloramiento de 


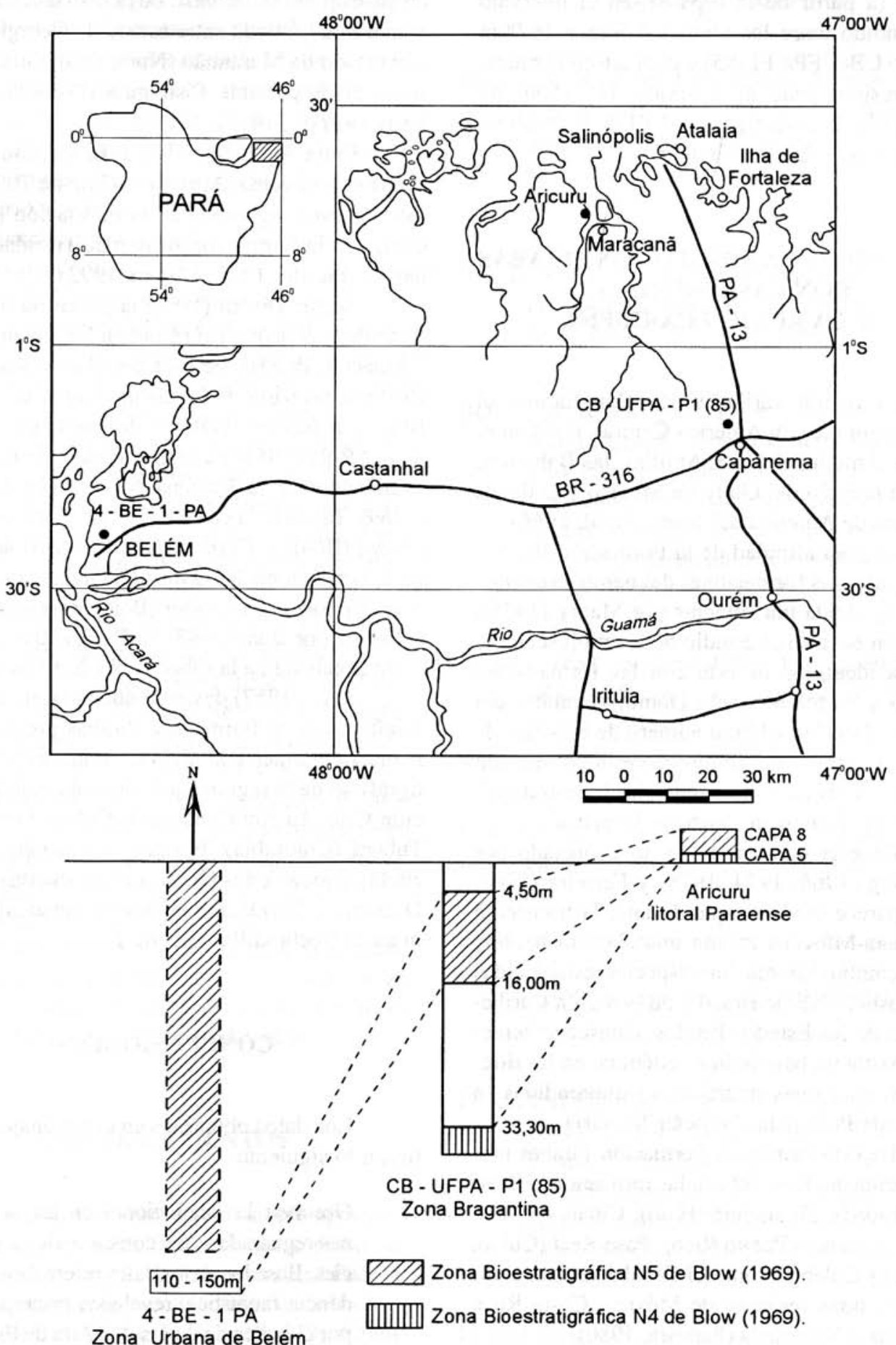

Fig. 5: Correlación bioestratigráfica entre los sedimentos de la Formación Pirabas de la región Bragantina, región urbana de Belém y el afloramiento de Aricuru. 
Aricuru (a partir de la capa 8), en el intervalo comprendido entre los niveles $4.50 \mathrm{~m}$ y $16.00 \mathrm{~m}$ del pozo CB-UFPa-PI (85) y también en la muestra correspondiente al intervalo $110-150 \mathrm{~m}$ de perforación de investigación 4-BE-1-PA realizada en la zona urbana de Belém.

\section{AFINIDAD DE LA FORMACIÓN PIRABAS CON LAS UNIDADES DE LA REGIÓN CARIBEÑA}

La región caribeña ocupaba durante el Mioceno un área en América Central, otras en el norte de América del Sur, Antillas, las Bahamas, parte de la costa del Golfo de México y la llanura costera de América del Norte (Bold, 1966).

La gran afinidad de la Formación Pirabas con las diversas formaciones del hemisfério Norte fue registrada inicialmente por Maury (1925), quien con base en el estudio de los moluscos admitió la identidad de ésta con las formaciones Chipola y Baitoa de Santo Domingo, ambas del Mioceno Inferior. El gran número de especies de moluscos comunes permitió que esta correlación fuera ampliada para otras unidades litoestratigráficas de la "Provincia Caribeña Terciaria".

De esta manera, según lo expresado por Woodring (1966, 1974, 1978) y Ferreira (1967, 1980) parece evidente que durante la transición Oligocena-Miocena existía una clara dispersión o intercambio de muchas especies establecidas en la costa N-NE de Brasil y en la región Caribeña y sur de los Estados Unidos. Consecuentemenete, existía un bajo índice endémico en las diferentes formaciones sincrónicas comprendidas en la llamada Provincia Caribeña Terciaria.

De esta manera la Formación Pirabas presenta también identidad con las formaciones Brasso (Trinidad), Thomonde (Haití), Cibao y Calcarias de Aymanas (Puerto Rico), Paso Real (Cuba), La Boca y Culebra (zona del Canal de Panamá) así como de otras regiones de México, Costa Rica, Colombia y Venezuela (Ferreira, 1980).

Entre los moluscos de la Formación Pirabas, que según Woodring (1966) sería el límite sur de la Provincia Caribeña Terciaria, el género de gastrópodo Orthaulax, cuya distribución horizontal está limitada entre estado de Georgia (EU) y el estado de Maranhão (Norte de Brasil), representa un importante fósil guía (Ferreira, 1967; Ferreira et al., 1973).

Entre los ostrácodos, esta similitud también es reconocida. Aproximadamente $70 \%$ de la ostracofauna registrada en la Formación Pirabas ocurre en la Formación Brasso de Trinidad y Tobago (Macedo, 1971; Távora, 1992).

Según Távora (1992) la presencia de Quadracythere brachypigaia Bold en los estratos de la Formación Pirabas, permite correlacionarla con la Biozona primaria Pokorniella "saginata" (sensu Bluter)/ P. laresensis (Bold), propuesta y reconocida por Bold (1983) en toda la región caribeña, extendiéndose de la Biozona N2 a la N5 de Blow (1969). También la coexistencia de Hermanites tschoppi (Bold) y Cushmanidea howei (Bold), permite correlacionar la Formación Pirabas con la subzona Hermanites tschoppi (Bold), propuesta y reconocida por Bold (1983) en Puerto Rico y Haití, correspondiendo a la subzona N4 de Blow (1969).

Petri (1957) describe que la fauna de foraminíferos de la Formación Pirabas presenta elementos comunes con algunas unidades litoestratigráficas de la región Caribeña, tal como Formación Cruz, El Abra y Guines (Cuba), Formación Tubará (Colombia), Formación Luciano (Venezuela), formaciones Gurabo y Las Puertas (Santo Domingo), Formación Bowden (Jamaica) y Formación Quebradillas (Puerto Rico).

\section{CONCLUSIONES}

Los datos obtenidos en este trabajo permitieron lo siguiente:

1. Observar las alteraciones en las asociaciones registradas, que corresponden a 8 biofacies. Basados en los datos referentes a la tendencia faunística, revelados principalmente por el índice de diversidad Alfa de Fisher (37) y la predominancia específica de los géneros más comunes (Ammonia, Amphistegina y Elphidium/Criboelphidium). 
Las cambios en las asociaciones permiten inferir una depositación de sedimentos de plataforma somera seguida por la instalación de un ambiente de lagunas con variaciones en la salinidad y la profundidad, donde la salinidad osciló entre normal a levemente hipersalina y la profundidad varió alrededor de $3 \mathrm{~m}$.

2. Correlacionar con base en los foraminíferos plantónicos:

- la base del afloramiento (capa 1) por la presencia de Globorotalia kugleri Bolli y Globigerinoides primordius Blow \& Banner con la zona bioestratigráfica N4 de Blow (1969);

- la capa 5, por la coexistencia de Globigerinoides primordius Blow \& Banner, Globorotalia kugleri Bolli y Globigerinoides altiaperturus Bolli al límite entre las zonas N4 y N5 de Blow (1969);

- $\quad$ los niveles superiores del afloramiento (a partir de la capa 8) por la presencia de Globigerinoides altiaperturus Bolli y Globigerinoides trilobus trilobus (Reuss) a la zona N5 de Blow (1969).

3. Ampliar el conocimiento de la distribución espacial de las zonas N4 y N5 de Blow (1969) en el área de la Formación Pirabas en el estado de Pará, así como establecer una correlación bioestratigráfica entre los puntos ya estudiados en la referida formación, localizados en el litoral Paraense, zona Bragantina y zona urbana de Belém, donde la zona N4 fue caracterizada en las dos primeras regiones fisiográficas y la zona N5 fue difinida en todas ellas.

\section{AGRADECIMIENTOS}

Los autores expresan un sincero agradecimiento a las siguintes instituciones y personas: Centro de Geociencias de la Universidad Federal do Pará, Departamento de Geologia de la Universidad Federal de Rio de Janeiro, Prof. Dr. Cândido Simões Ferreira, Prof. Dr. Maurício da Silva Borges, Prof.Dr. Werner Truckenbrodt y Prof. Cláudio Fabian Szlafsztein.

\section{REFERENCIAS}

BLOW, W.H., 1969: Late Middle Eocene to Recent Planktonic Foraminiferal Biostratigraphy. - International Conference on Planktonic Microfossils, Geneve, 1: 199475, 43figs., 54 plates.

BOLD, W. A. van den, 1966: Ostracodes Zones in Caribbean Miocene. - Am. Assoc. Pet. Geol. Bull., 50(5): 1029-1031.

BOLD, W.A.van den, 1983: Shallow-Marine Biostratigraphic Zonation in the Caribbean Post-Eocene. - En: Maddocks, R.F. (ed.): Applications of Ostracoda, Proceedings of the Eight International Symposium on Ostracoda, Departmant of Geosciences, University of Houston, Houston: 400-416.

BOLLI, H.M., SAUNDERS, J.B. \& PERCHNIELSEN, K.,1989: Plankton Stratigraphy. - Vol. 1 y 2, University Press, Cambridge.

BOLTOVSKOY, E., 1965: Los Foraminiferos Recientes.- 510 págs.; EUDEBA, Buenos Aires.

FERNANDES, J.M.G., 1988: Bioestratigrafia da Formação Pirabas, Estado do Pará. - An. XXXV Congr. Brasileiro Geol., Belém, 6: 2376-2382.

FERNANDES, J.M.G. \& TÁVORA, V.A., 1990: Estudo dos Foraminíferos da Formação Pirabas Procedentes do Furo CB-UFPaP1 (85), Município de Capanema, Estado do Pará. - An. XXXVI Congr. Brasileiro Geol., Natal, 1: 470-475.

FERREIRA, C.S., 1966: Características Lito-Paleontológicas da Formação Pirabas, Estado do Pará. - Av. Div. Geol. Mineral., Rio de Janeiro, 41: 101-111. 
FERREIRA, C.S., 1967: Contribuição à Paleontologia do Estado do Pará. O Gênero Orthaulax Gabb, 1872 na Formação Pirabas. X. (Mollusca- Gastropoda). - At. Simp. Biota Amaz., Belém, 1: 169-185.

FERREIRA, C.S., 1980: Correlação da Formação Pirabas (Mioceno Inferior), N e NE do Brasil, com as Formações Chipola e Tampa da Península da Florida, USA. - An. I Congr. Latinoamericano Paleont., Buenos Aires, 3:49-55.

FERREIRA, C.S., GONZALEZ, B.B. \& FRANCISCO, B.H.R., 1973: Ocorrência da Formação Pirabas (Mioceno Inferior) na Bacia de Barreirinhas, Maranhão. - Rer. Bras.Geoc., 3(1): 60-67.

GIBBSON, G.G. \& HILL, E.F., 1992: Species Dominance and Equitability: Patterns in Cenozoic of Eastern North America. - J. Foraminiferal Res., 22 (1): 34-51.

LOEBLICH, A.R. \& TAPPAN, H., 1964: Protist 2- Sarcodina. - En: Moore, R.C. (ed.), Treatise on Invertebrate Paleontology, Part C, The Geological Society of America, New York, 2v., 900 págs., 653figs.

MACEDO, A.C.M., 1971: Considerações Sobre os Ostracodes do Mioceno Marinho do Estado do Pará. - An. Acad. Bras. Cien., 43(suplemento): 523-528.

MAURY, C.J., 1925: Fósseis Terciários do Brasil com Descrição de Novas Formas Cretáceas. - Monog. Serv. Geol. Mineral. Brasil, Rio de Janeiro, 4: 1-665.

MOURA, J.A. \& ABREU, W.S., 1980: Contribuição ao Estudo Paleoecológico da Bacia da Foz do Rio Amazonas. - Rev. Bras. Geoc., 10: 73-88.

MURRAY, J.W., 1973: Distribution and Ecology of Living Benthonic Foraminiferids. - 274 págs.; Crane Russak e Co., New York.

PETRI, S., 1957: Foraminíferos Miocênicos da Formação Pirabas. - Bol. Fac. Filos. Ciênc. Letr., Univ. São Paulo, São Paulo, 216: 1-79.

STAINFORTH, R.M., LUTHERBACKER, H., BEARD, J.H. \& JEFFORDS, R.M., 1975: Cenozoic Planktonic Foraminiferal Zonation and Characteristics Index Forms. University of Kansas, Paleontological Contributions, Kansas, 62: 1-425.

TÁVORA, V.A., 1992: Ostracodes da Formação Pirabas (Mioceno Inferior) no Estado do Pará, Brasil. - Inst. Geoc., Univ. Fed. Rio de Janeiro, Dissertação de Mestrado, 88págs.

WALTON, R., 1964: Recent Foraminiferal Ecology and Paleoecology. - En: IMBRIE, J. \& NEWELL, N. (eds.): Approaches to Paleoecology: 151-273; New York.

WOODRING, W.P., 1966: The Panama Land Bridge as a Sea Barrier. - Am. Philos. Soc. Proc., 110: 425-433.

WOODRING, W.P., 1974: Affinities of Miocene Marine Molluscan Faunas on Pacific Side of Central America. - Public. Geol. del ICAITI, 4: 179-188.

WOODRING, W.P., 1978: Conexiones Terrestres entre Norte y Sudamerica. - Univ. Nac. Autonoma Mexico, Inst. Geol., 101: 153-165. 